\title{
Xu's Designs and Parameters for Sealing Elements
}

\author{
Xu Changxiang \\ Zhejiang China Valve Co. Ltd., Wenzhou, Zhejiang, 325024, China \\ Email address: \\ xchx9763@sina.com
}

\section{To cite this article:}

Xu Changxiang. Xu's Designs and Parameters for Sealing Elements. International Journal of Energy and Power Engineering. Special Issue: Xu's Sealing and Flowing Theories of Fluids. Vol. 5, No. 4-1, 2016, pp. 35-42. doi: 10.11648/j.ijepe.s.2016050401.15

Received: June 22, 2016; Accepted: June 25, 2016; Published: August 24, 2016

\begin{abstract}
Any leak-free achievement needs a sealing surface that has microcosmic irregularities fully complementary to a surface to be sealed and that can be qualified only by compressive working or installing in position. ASME Code mistakes the working stress needed to result in a qualified gasket in installation for the stress needed to enable a qualified gasket to function well or maintain its leak-free connection, and only considers the influence of the sealing material's strength but never its elasticity on sealing. Undoubtedly, ASME Code's leak-free Maintenance Factor $m$ and Minimum Necessary Sealing Stress $y$ of gaskets are both wrong in values. Similarly, neither is the gasket parameters system substituted for the gasket factors $m$ and $y$ by PVRC and EN 13555. Actually, the stronger the strength and the elasticity of a sealing contact layer, the more difficult for its sealing element to create or maintain a fully deformed contact; whereas the stronger the strength and the elasticity of a contact layer substrate, the easier for its sealing element to create or maintain a fully deformed contact; i.e. the index used to measure the difficulty for a sealing element to create or maintain a leak-free connection is its sealing difficulty factor $m_{l}=$ its contact's elastic modulus $E_{c} /$ its substrate's elastic modulus $E_{s}$; so it is the most difficult for a rubber element to create or maintain a leak-free connection because its $m_{l} \equiv 1$, the easiest for a grease coating because its $m_{l}=0$, and easier for the other sealing elements than for a rubber element because their $m_{l}$ can be made smaller than 1 (or $m_{l}<1$ ) by designing and coating.
\end{abstract}

Keywords: Minimum necessary sealing stress $y$, Sealing difficulty factor $m_{l}$, Leak-Free maintenance factor $m_{2}$

\section{Gasket Factors $\boldsymbol{m}$ and $\boldsymbol{y}$ of ASME Code}

Two gasket factors $m$ and $y$ used to calculate flange bolt loads are defined in the ASME Boiler and Pressure Vessel Code Division 1, Section VIII, Appendix 2 (hereafter called ASME Code) ${ }^{[1]}$.

The leak-free connection of two ASME flanges, as shown in Fig.1, is achieved by a jointing gasket between two end faces and some gasket-loading bolts, and hence is a pressure-tight connection, and the more tightened the bolts, the better the sealing performance within the allowable strength of gasket material. As shown in Fig.2, the two flanges have a wide touch width $b$ due to no bending before tightened, a narrowed touch width $b$ due to some bending after tightened, and a more narrowed touch width $\mathrm{b}$ due to more bending after pressurized. Generally speaking, tightening for assembly is causing a gasket to be loaded or resulting in a gasket increasing its sealing stress, and fluid pressure is causing a gasket to be unloaded or resulting in a gasket decreasing its sealing stress.

ASME Gasket's leak-free Maintenance Factor $m$ (see Fig.3), $m=\left(W-A_{2} p\right) / A_{l} p$, is a factor that provides the additional preload needed in the flange fasteners to maintain a compressive load on a gasket after a fluid pressure is applied to a joint, where $W=$ Total fastener load, $A_{l}=$ Gasket's sealing contact area, $A_{2}=$ Gasket's inner circular area, $p=$ Fluid pressure ${ }^{[2]}$.

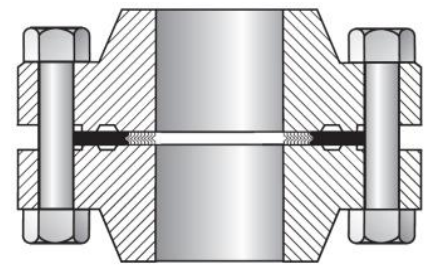

Fig.1 ASME Bolted Flanges
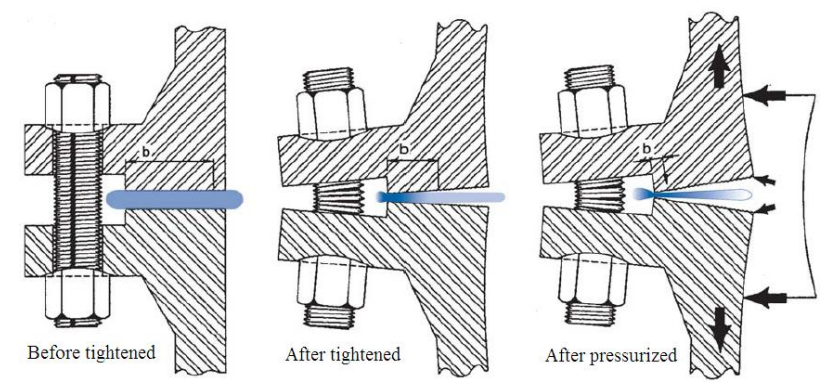

Fig.2 ASME Flange Rotation ${ }^{[2]}$ 
So it is undoubted that $\left(W-A_{2} p\right)$ is the force that can create the sealing stress on the sealing surface at a fluid pressure, and $A_{l} p$ is the unsealing force of leaking fluid on the sealing surface.

ASME Gasket's Minimum Necessary Sealing Stress $y, y=$ $W / A_{l}$, is the minimum compressive stress on the contact area of the gasket that is required to provide a leak-free connection at atmospheric pressure ${ }^{[2]}$.

Any leak-free achievement needs to seat a sealing surface into irregularities on a surface to be sealed, whereas any surface of rubber, because it is both the softest and the most elastic material, is the most easily stretched to be away from the pit bottom by the irregular pit edge on the being sealed surface; and hence rubber is the most difficult material for creating a leak-free connection or its minimum necessary sealing stress $y$ is the greatest in all the materials. Any full leaking is a process for a leaking fluid on the sealing contact surfaces to output a contact-separating force whose limit is equal to "contact area $x$ fluid pressure", so that the stress $y$ of rubber at least includes a component withstanding an atmospheric pressure $\left(0.1 \mathrm{MP}_{\mathrm{a}}\right)$ or never equals zero. However, ASME Code has deemed the stress $y$ of rubber to be 0 and the stress $y$ of the other materials to be $180(2 m-1)^{2}$, where $m$ $=0 \sim 6.5$; i.e. the values of $m$ and $y$ have serious problems in ASME Code. Incorrect as the values of gasket factors $m$ and $y$ of ASME Code are, their basic definitions are very scientific.

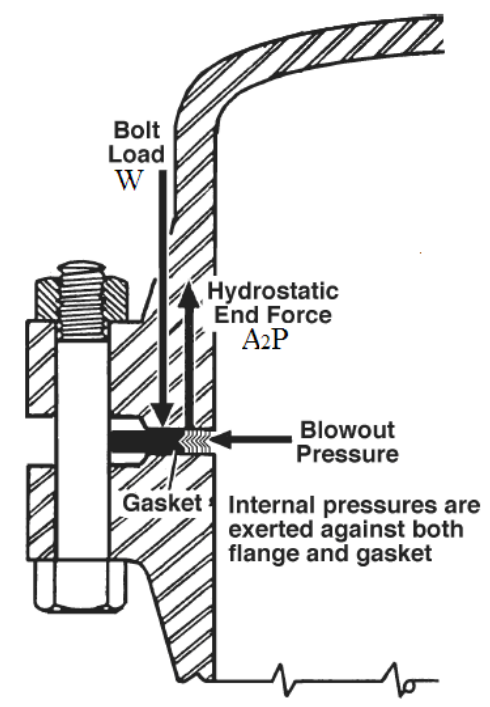

Fig.3 Forces Acting on an ASME Gasket ${ }^{[3]}$

\section{Gasket Parameters to Be Substituted for ASME Code's $\boldsymbol{m}$ and $\boldsymbol{y}$}

PVRC suggests that ROTT gasket constants $G_{b}$, a and $G_{s}$ are substituted for gasket factors $m$ and $y$ of ASME Code ${ }^{[4-6,9]}$, and EN 13555 substitutes gasket parameters $Q_{M I N, L}, Q_{S M I N, L}$ and

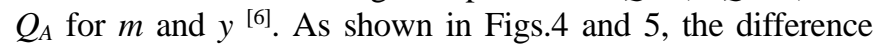
between the constants and the parameters is their expressing ways or expressed aspects.

In Fig. 4 for ROTT gasket constant curves consisting of a gasket-loading line in assembly and a gasket-unloading line in service, $G_{b}$ stands for the stress at loading a gasket up to its given sealing start in assembly, $a$ for the slope of the assembly line, $Q_{A}$ for the gasket stress at the assembling finish, $Q_{\text {MASS }}$ for the ultimate strength of gasket material, and $G_{s}$ for the stress at unloading a gasket up to its given sealing finish by a fluid pressure in service. As for a gasket up to a required tightness, why is its stress $Q_{S M I N, L}$ required in service smaller than its stress $Q_{M I N, L}$ required in assembly? It is because any gasket unassembled into position is only a preprocessed semi-finished gasket whose shape does not conform to the sealing requirements and shall need such a deforming assembly in position as to be able to work it into a qualified gasket with fully deformed contact and support functions, whereas a qualified gasket in service does not need that deforming stress; i.e. $Q_{M I N, L}$ is the stress needed to work a preprocessed gasket into a qualified gasket in assembly, and $Q_{S M I N, L}$, the stress needed for a qualified gasket to maintain a given tightness in service. In fact, it is when the unloading of a gasket causes its spring back to be unable to maintain its tight contact that its sealing will fail, and its leaking resistance depends on its design, preprocessing and assembling; or the leak-free performance of a gasket depends on its qualities of design and manufacture including assembly. However, PVRC and EN 13555 mistake the gasket-working stress for the gasket's necessary sealing stress and always focus on leaking during an unqualified assembling or manufacturing process of badly-designed gaskets.

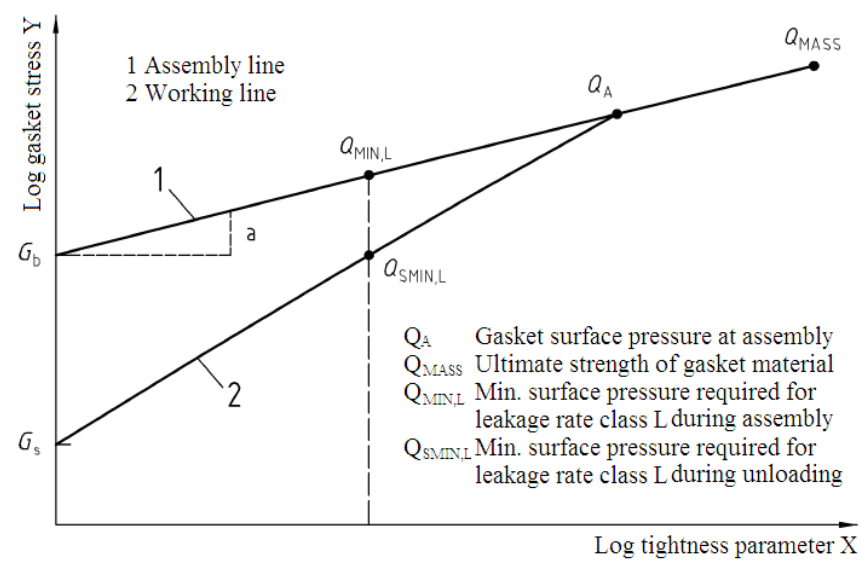

Fig.4 PVRC's ROTT Gasket Constants (for all internal pressures)

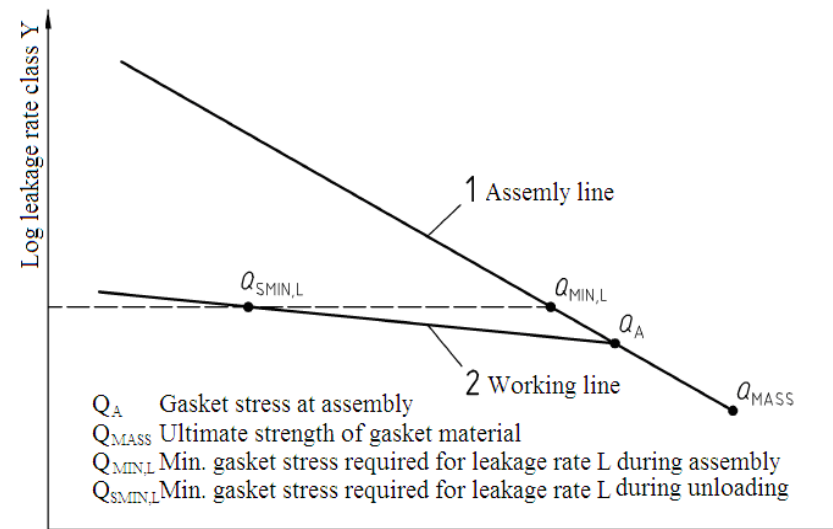

Gasket stress X

Fig.5 EN 13555's Gasket Parameters (for a given internal pressure) 


\section{Xu's Designs and Parameters of Seal- ing Elements}

As shown in Fig.6, two microsawtooth rings (04), a metallic (02) or nonmetallic (03) ring and grease coatings on the opposing faces are typical Xu's sealing elements that are used to form three independent seals of Xu's bolted flange joint. A macrosawtooth ring (05) is an indispensable design for ensuring that the sealing elements are parallel or circumferentially uniformly compressed by the two flange opposing faces ${ }^{[7]}$. Typical Xu's parameters of sealing elements, as shown in Fig.7, are the parameters of theoretical lines loading and unloading Xu's steel microsawtooth rings $S_{-1}$ and rectangular rings $S_{-2}$ with or without soft coatings $S_{\mathrm{cc}}$ as well as rubber rectangular rings $\mathrm{S}_{-3}$ and grease coatings $\mathrm{S}_{-4} G_{b 1}$ is the stress where the microsawtooth ring top $S_{\mathrm{c} 1}$ is just compressed into irregular depressions on its opposing face; $S_{a l}^{\prime}$, the stress where the soft coating $S_{\mathrm{cc}}$ is just compressed into a thin film that has the same strength as its substrate, and may not be on the Y-axis; and $S_{a l}$, the stress where the substrate of microsawtooths or coatings is

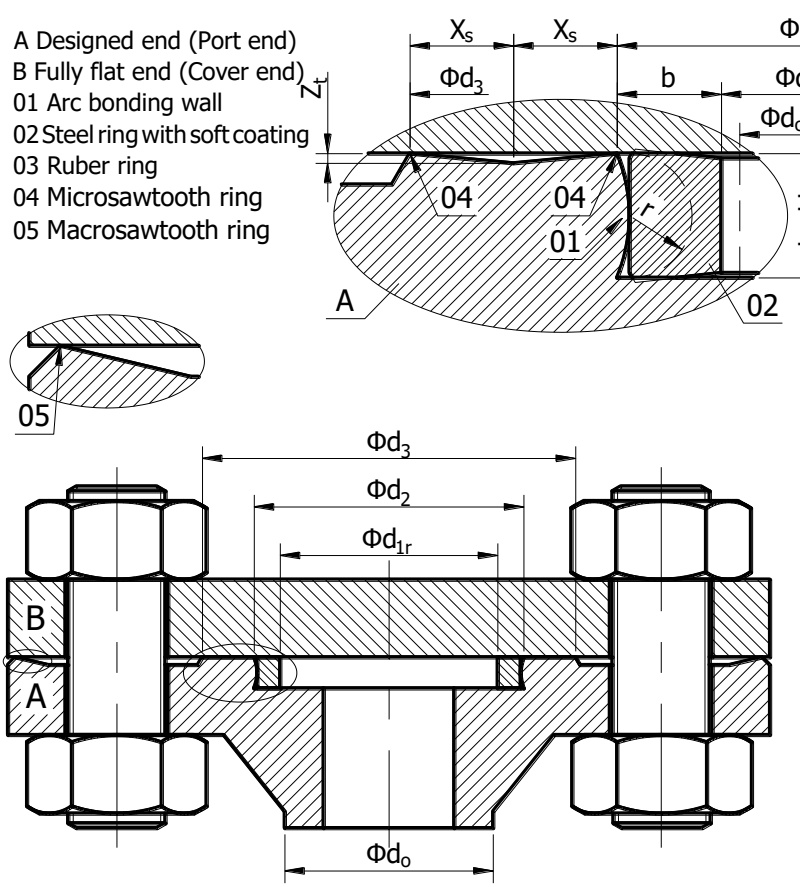

compressed up to its full elastic deformation, and also the finish where the microsawtooth ring ridge has been solely compressed. $G_{b 2}$ is the stress where the end ridge top $\mathrm{S}_{\mathrm{c} 2}$ of metallic rectangular rings is just compressed into irregular depressions on its face to be sealed; $S_{a 2}^{\prime}$, the stress where the soft coating $\mathrm{S}_{\mathrm{cc}}$ is just compressed into a thin film that has the same strength as its substrate, and may not be on the Y-axis; and $S_{a 2}$, the stress where the rectangular ring body is compressed up to its full elastic deformation, and also the finish where the rectangular ring has been solely compressed. In other words, $G_{b}$. is the stress required to just work a machined sealing element into a qualified seal in position, not the minimum sealing stress needed to maintain a leak-free connection. Only $y$-is the minimum necessary sealing stress required to maintain a fully leak-free connection at atmospheric temperature and pressure and equal to the compressive stress on the contact surface at the moment just before the sealing contact is circumferentially uniformly unloaded up to its leakage in the original position where it was circumferentially uniformly loaded up to its fully leak-free state. $\Phi \mathrm{d}_{2}$

\section{$\Phi d_{1 \mathrm{r}}$ $\Phi d_{0}$$$
\text { 魚 }
$$
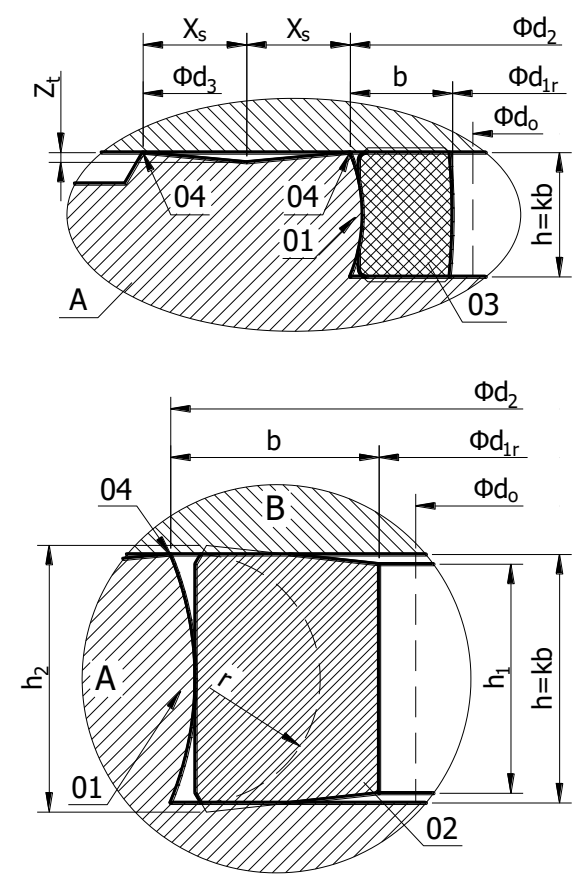

Fig.6 Xu's Designs for Sealing Elements

A leak-free connection results from loading a sealing contact layer first up to its fully deformed contact and then up to its fully tight contact, or any leak-free achievement needs first to create a fully deformed contact that can seat a sealing surface into irregularities on a surface to be sealed and then to create a fully tight contact that can resist a seepage through the sealing contact, and any full seepage or leaking is a process for a leaking fluid on the two contact surfaces to output a contact-separating force whose limit equals "contact area $\mathrm{x}$ fluid pressure". Hence, the minimum stress needed to resist an atmospheric seepage through the contact is the minimum necessary sealing stress $y$ for a seal, and the contact whose stress is up to the minimum necessary sealing stress $y$ is called the minimum necessary tight contact. In order to achieve a fully deformed contact without any flaw, it is better to load the sealing contact layer up to its fully yielded deformation. In order to achieve a fully tight contact, it is needed to load the sealing contact layer substrate up to its fully elastic deformation to provide a fully powerful support for the contact. Therefore, as shown in Fig.7, the contact stress at loading a sealing contact just up to its fully deformed contact without any flaw at atmospheric pressure is the flaw-blocking or sealing beginning stress $G_{b}$; the assembled stress $S_{a \text { - }}$ at loading the substrate up to its fully elastic deformation shall approach the yield strength $S_{e}$ - of the substrate material; the contact stress at unloading a fully leak-free contact just up to being leaving its fully tight contact is the leakage-starting stress or the minimum necessary sealing stress $y$; the contact stress at further unloading the contact just 
up to being leaving its fully deformed contact is the flaw-reproducing or sealing-stopping stress $G_{s-}$; and $y_{-}=y_{e_{-}}+\mathrm{y}_{\mathrm{a}}$, where $y_{e}$ is the component for loading the sealing contact just up to restoring its full deformation on unloading line, and $\mathrm{y}_{\mathrm{a}}=$
$0.1 \mathrm{MP}_{\mathrm{a}}$ is the component for loading the fully deformed contact just up to restoring its minimum necessary tight contact that can fully resist the atmospheric seepage through the contact on unloading line.

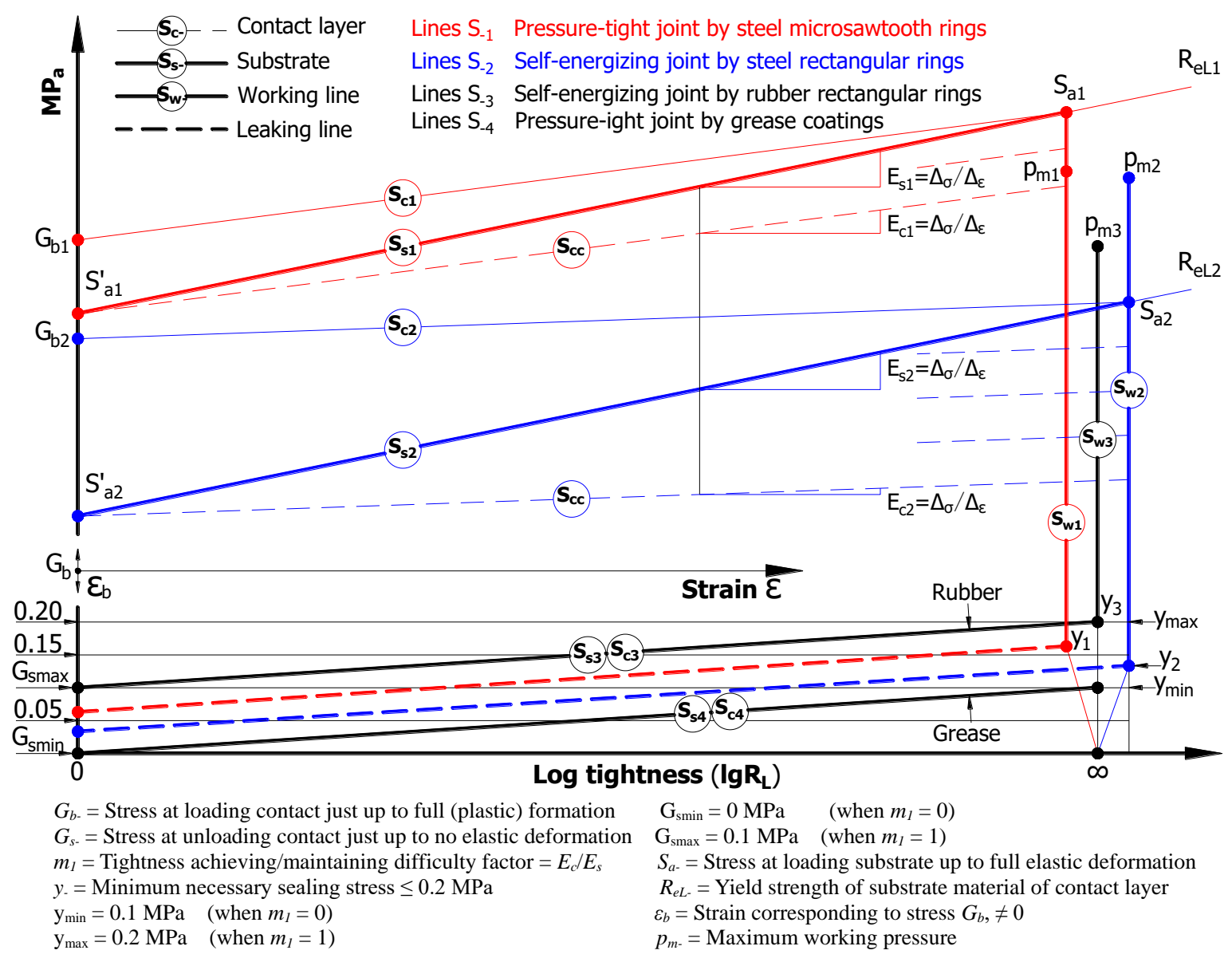

Fig. 7 Xu's Parameters for Sealing Elements

The stronger the strength and the elasticity of a sealing contact layer, the more difficult for its sealing element to create or maintain a fully deformed contact; whereas the stronger the strength and the elasticity of a contact layer substrate, the easier for its sealing element to create or maintain a fully deformed contact ${ }^{[8]}$; i.e. the difficulty for a sealing element to create or maintain a leak-free joint is determined by its sealing difficulty factor $m_{l}, m_{l}$ = contact layer's elastic modulus $E_{c} /$ substrate's elastic modulus $E_{s}$; or the greater the value of $m_{l}=E_{c} / E_{s}$ for a sealing element, the more difficult for it to create or maintain a leak-free joint, and also the greater the values of the sealing-stopping stress $G_{s}$ and the minimum necessary sealing stress $y$. As shown in Fig.8, the contact layer's elastic modulus $\left(E_{c}\right)$ for a general sealing element can be the residual elastic modulus of its yielded material, and the substrate's elastic modulus $\left(E_{s}\right)$, the elastic modulus of its material before yielded; and for a rubber sealing element without yielding, $E_{c}=E_{s}$. Therefore, it is the easiest for a grease coating to create a leak-free joint whose $E_{c}=0$ or $m_{l}=0$; it is the most difficult for a rubber sealing element to do whose $E_{c}=E_{s}$ or $m_{l}=1$; and it is far easier than for a rubber sealing element for any other sealing element such as a metal element to do whose contact layer can be weakened by designing or coating to cause its $E_{c}<E_{s}$ or $m_{l}<1$.

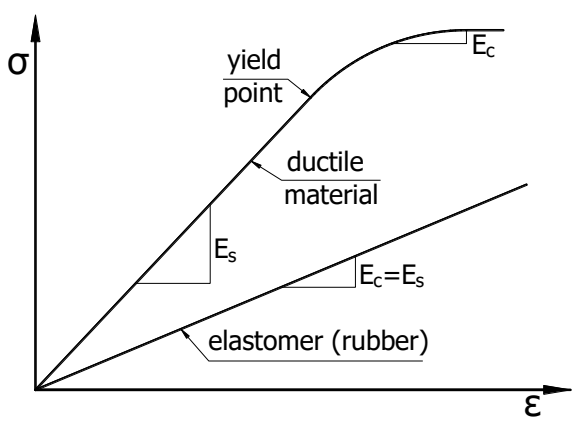

Fig. 8 Xu's sealing difficulty factor $m_{l}=E_{c} / E_{S}$

From the fact that grease can be easily deformed and not return to its original shape at atmospheric pressure, it can be seen that the internal pressure caused by the elastic strength of a free grease body is $0 \mathrm{MP}_{\mathrm{a}}$; i.e. for a grease coating, $G_{b}=G_{s}=0$, or $y_{e}=0$. From the fact that rubber can be easily deformed and return to its original shape at atmospheric pressure, it can be seen that the internal pressure caused by the elastic strength of a free rubber body is $0.1 \mathrm{MP}_{\mathrm{a}}$; i.e. for a rubber element, $G_{b}=$ $G_{s}=0.1 \mathrm{MP}_{\mathrm{a}}$, or $y_{e}=0.1 \mathrm{MP}_{\mathrm{a}}$. Therefore, as to any qualified sealing element, its lower limit of sealing-stopping stresses 
$\mathrm{G}_{\mathrm{smin}}=0$ (equals $G_{b}$ value of grease coatings), its upper limit of sealing-stopping stresses $\mathrm{G}_{\text {smax }}=0.1 \mathrm{MP}_{\mathrm{a}}$ (equals $G_{b}$ value of rubber elements), its lower limit of minimum necessary sealing stresses $\mathrm{y}_{\min }=0.1 \mathrm{MP}_{\mathrm{a}}$ (equals $y$ value of grease coatings), its upper limit of minimum necessary sealing stresses $\mathrm{y}_{\max }=0.2 \mathrm{MP}_{\mathrm{a}}$ (equals $y$ value of rubber elements), and $\mathrm{G}_{\mathrm{smax}}$ $\mathrm{G}_{\text {smin }}=\mathrm{y}_{\max }-\mathrm{y}_{\min }=y-G_{s}=0.1 \mathrm{MP}_{\mathrm{a}}$ (standard atmospheric pressure); or any sealing element is unqualified whose minimum necessary sealing stress $y$ is more than $0.2 \mathrm{MP}_{\mathrm{a}}$.

Any leaking through a sealing contact starts at the moment when the contact-tightening stress is less than the fluid pressure, and tries to cause the contact-tightening stress to be counteracted and cause the fluid to be decompressed up to atmospheric pressure by fluid separation of contact. Because the contact separating force increases as the seepage-wetted area increases before a full leaking and lowers as the fluid pressure lowers after a full leaking, thus the moment when the contact-separating force is to be up to the limit of "contact area $x$ fluid pressure" is the moment when the contact-tightening force and stress are to be thoroughly counteracted and cause a full leakage or a full decompression of fluid. If at this moment adding a tightening stress not less than atmospheric pressure to the contact surface, the contact tightening stress will not be less than the fluid pressure that is lowering up to atmospheric pressure and can prevent the sealing contact from leaving its fully tight state. In other words,

- any sealing contact can never be unloaded to leave its fully tight state and, even if separated by a disturbing force for a moment, will return to its fully tight state right after a full leaking as long as the contact-tightening force is positively greater than the contact separating force limit of "contact area x fluid pressure";

- the contact stress that can resist an atmospheric seepage through the contact is the minimum necessary sealing stress $y$ for a seal;

- any seal can never leak as long as its sealing stress can be always not less than its minimum necessary sealing stress $y$ or any leaking cannot happen until the sealing stress is less than the stress $y$, and

- any seal shall be designed to make its minimum necessary sealing stress $y$ not more than $0.2 \mathrm{MP}_{\mathrm{a}}$.

Therefore, according to the foregoing definition of the minimum necessary sealing stress $y$ and the definition of the leak-free maintenance factor $m_{2}, m_{2}=$ joint's sealing actuation force $F_{s}$ /joint's unsealing actuation force $F_{u}$, for a sealing joint, where $F_{s}=$ the fastener or fluid actuation force causing a tight contact and $F_{u}=$ contact separating force limit $=p A_{u}$, the condition for a pressure-tight joint to maintain its leak-free contact is:

$\left(F_{s}-F_{u}\right) \geq y A_{u} \rightarrow\left(m_{2} p A_{u}-p A_{u}\right) \geq y A_{u} \rightarrow m_{2} \geq(1+y / p) ;$ and the condition for a self-sealing joint to maintain its leak-free contact is:

$\left(F_{s}-F_{u}\right) \geq y A_{u} \rightarrow\left(p A_{s}-p A_{u}\right) \geq y A_{u} \rightarrow m_{2}=A_{s} / A_{u} \geq(1+y / p) ;$ where $y=$ minimum necessary sealing stress, $p=$ fluid pressure, $A_{s}=$ fluid pressure actuation area of a self-sealing ring, $A_{u}=$ sealing contact area of a sealing element.

Given that $y \leq 0.2 \mathrm{MP}_{\mathrm{a}}$, thus, if any joint whose pressure rating $p_{n} \leq 1 \mathrm{MP}_{\mathrm{a}}$ can be designed according to $p_{n}=1 \mathrm{MP}_{\mathrm{a}}$, then the condition for any joint to be up to resisting a seepage through the sealing contact at an ultimate pressure of $p_{b}=4 p_{n}$ $=$ ultimate strength of its jointed body, whether it is a pressure-tight joint or a self-sealing joint, can be its leak-free maintenance factor $m_{2} \geq\left(1+y / p_{b}\right)=(1+0.2 / 4)=1, m_{2}$ being required for $F_{s}=m_{2} F_{u}$; i.e. the total fastener load $\left(F_{\Sigma}\right)$ needed for any sealing joint is $F_{\Sigma} \geq \pi p_{n} d_{2}^{2}$, where $p_{n}=$ pressure rating, and $d_{2}=$ external diameter of a sealing ring. For example, as for the self-sealing joint in Fig.6, $F_{\Sigma}=F_{s}+p_{b} A_{l r}=m_{2} F_{u}+$ $p_{b} A_{l r}=m_{2} p_{b} A_{u}+p_{b} A_{l r} \geq p_{b}\left(A_{u}+A_{l r}\right)=p_{b} A_{2}=\pi p_{n} d_{2}^{2}$.

As to the metallic rectangular self-sealing ring (02) shown in Fig. 6 that has a radial clearance between its body and its bonding wall (01) and is designed to outwards acuminate or dwindle its both end walls according to "height $\left(h_{2}\right)$ between its two end ridge tops > depth $(h)$ of its cavity > height $\left(h_{l}\right)$ between its two end ridge bottoms", it can have "its end area $<$ its body area < its cavity wall area" in cross sections, and hence, as long as it has an enough compressing force in the first installation, it must first have such a wholly outward bulged elastic Poisson's deformation enough to make it tight against the middle of its bonding wall, and then further against the middle have such a locally outward bulged severe-to-mild plastic Poisson's deformation in the two parts from its end ridge top to its end ridge bottom, and such a locally outward bulged severe-to-mild elastic Poisson's deformation in the two parts from its end ridge bottom to its middle as to enable it to rotate in its cavity but not to be taken out of its cavity by fingers after decompressed; as long as it has an enough uniform circumferential compressing force in the repeated installation, its body has a being compressed allowance enough to repeatedly provide the full plastic deformation for its sealing contact surfaces; and as long as its wall is thinner than the pipe wall to be connected, it will first have two wedged sealing deformations caused by its wall's rotation with $r$ as the rotating radius in the connecting system as an internal fluid pressure rises after well installed. In other words, as to the metallic rectangular self-sealing ring (02) shown in Fig.6, its first installation and its each repeated installation can both ensure its sealing contact surface a full plastic deformation and its contact surface substrate a full elastic deformation or ensure its sealing difficulty factor $m_{l}$ is less than 1 or than the factor $m_{l}$ of rubber elements or ensure its minimum necessary sealing stress $y$ is less than $0.2 \mathrm{MPa}_{\mathrm{a}}$ or than the stress $y$ of rubber elements as long as it has a compressing force great and uniform enough for each installation, and it always has a unique deforming sign that it can be brought into and out of its cavity before first compressed and can rotate in its cavity but not be taken out of its cavity by fingers after compressed and decompressed ${ }^{[7]}$.

In sum, as long as the contact rigidity of the sealing surface is less than the support rigidity of the sealing surface or "the resistance of the sealing surface to compression < the resistance of the sealing surface substrate to compression < the tensile strength of the jointed body" or as long as it is ensured that the sealing difficulty factor is less than one, there is absolutely no sealing element that cannot be loaded up to a fully deformed and tight contact and also no condition that can cause its sealing contact surface to have a contact stress less 
than its minimum necessary sealing stress $y$ and be away from its fully tight contact when its total fastener load is designed according to matching 4 times pressure rating that can cause its jointed body to break or according to ensuring that its leak-free maintenance factor $m_{2} \geq 1$. In other words, as long as designed according to its sealing difficulty factor $m_{l}<1$ and its leak-free maintenance factor $m_{2} \geq 1$ and assembled to a full qualified deformation, neither self-sealing element nor pressure-tight element can leak until its jointed body breaks, which is as shown as by the vertical working lines $S_{w}$ in Fig.7.

In the prior art, there is neither such a designing concept or method resulting in a both soft and strong seal nor such a tightening concept or method resulting in an assembled seal with a $\mu \mathrm{m}$ level of circumferentially uniform contact (see annex A) that spiral wound gaskets, Kammprofile gaskets and solid metal core gaskets that are inadvertently in best accordance with the designing concept resulting in a both soft and strong seal cannot yet have a fully strong support and resilience in each orientation as their material density and their tightening load cannot be fully uniform in circumferential direction. Naturally, in addition to the leaking through gasket material and between spiral layers, there is no leak-free pressure-tight joint in the prior art or it is absolute that any gasket joint is leaking in the ROTT method. Given that the working line in ROTT method is almost a leaked line through the start $\left(S_{a}\right)$ of Xu's working segment without any leakage and the finish $\left(G_{s}\right)$ of Xu's leaking segment without any contact flaw, the sealing beginning $\left(G_{b}\right)$ and the unsealing start $\left(G_{s}\right)$ of Xu's sealing element are basically corresponding to ROTT constants $G_{b}$ and $G_{s}$. Since an enormous gasket working stress component is included in ROTT's minimum sealing stress required for a leakage class $L$, the ROTT's calculation stress and the actual stress for a leakage class $L$ differ at least by dozens of times ${ }^{[9]}$. However, the crush resistance tightness tests on a Selco gasket have almost definitely proved that a pressure-tight joint qualified in both design and assembly can work without leaking ${ }^{[10]}$ (see annex A). Therefore, the leaked working line defined in ROTT method is wrong and the Xu's leak-free working line for sealing elements is correct.

The minimum necessary sealing stress $y=0.2 \mathrm{MP}_{\mathrm{a}}$ for a rubber sealing element not only can be as inferred as the above mentioned but also can be fully proved by Xu's stress calculating formula for O-rings ${ }^{[7]}$ and Parker's O-ring leak rate curve ${ }^{[11]}$. The simplest method proving that $y$ is less than $0.2 \mathrm{MP}_{\mathrm{a}}$ for any other sealing element is that a self-sealing joint can pass any pressure test up to burst pressures when loosened to a finger-tightened state in the original position after tightened to a fully deformed state, and a pressure-tight joint by microsawtooth rings, being pressurized by a certain pressure source, can over and over suddenly leak without any slow leakage when circumferentially uniformly tightened by wrench to a properly tight state.

\section{Conclusions}

Theoretically, any sealing element shall be designed to have a soft \& inelastic contact layer and a strong \& elastic contact layer substrate, and assembled to form both a fully (plastically) deformed contact and a fully elastically deformed support, for any leak-free achievement needs a sealing surface that has microcosmic irregularities fully complementary to a surface to be sealed and that can be qualified only by compressive working or installing in position.

The sealing performance of a gasket originates in its whole qualities of design and manufacture including assembly. To deform a semi-finished gasket into a fully qualified gasket in assembly, its system design shall conform to "total tensile capacity or area of its fastener $>$ tensile capacity or area of its connected pipe $>$ cross sectional area of its body $>$ cross sectional area of its end" and its processes before assembly shall conform to its designs. The final criterion for a qualified gasket is if its assembly can cause it to have a minimum necessary sealing stress y less than $0.2 \mathrm{MP}_{\mathrm{a}}$, but never does any leakage test on its assembly that is only a manufacturing process according to ROTT method and EN 13555.

Note: This paper was initially published in Chinese magazine of Petro-Chemical Equipment, 2013, Volume 42 (3): 58-63.

\section{References}

[1] ASME Boiler and Pressure Vessel Code Division 1, Section VIII, Appendix 2 Rules for Bolted Flange Connections with Ring Type Gaskets [S].

[2] Garlock Technical Manual (GMG 1-11).

http://www.garlock.com/en/

http://www.novatec.cr/Garlock-Empaq-Sellos/Juntas_laminados /Graphonic_Manual_tecnico.pdf

[3] Garlock Metallic Gasket Catalog (GMG 1-1) http://www.garlock.com/en/product/graphonic-metallic-gasket http://galloup.com/downloads/garlock_metallic_gasket_catalog.pdf

[4] ASTM WK10193 New Recommended Practice for Gasket Constants for Bolted Joint Design [S].

[5] CETIM Report of ROTT Tests on ASME B16.20 SRI http://www.leadergasket.sk/zertifikate/ROTT_tests_on_ASME _B16.20_SRI.pdf

[6] EN 13555 Flanges and Their Joints - Gasket Parameters and Test Procedures Relevant to the Design Rules for Gasketed Circular Flange Connections [S].

[7] XU Changxiang. XU's Sealing Theory and Rectangular \& O-Shaped Ring Seals [J].PETRO-CHEMICAL EQUIPMENT, 2013,42 (2): 75-85.

[8] Garlock Helicoflex Master Catalog Metric. http://www.techneticsgroup.com/bin/371.pdf.

[9] Applications Engineering Department. Gasket Constants for the Layman, Garlock Technical Bulletin [OL]. 2004-09-08. http://www.garlock.com/eng_tools/gasketing/designInformation /Gasket\%20Constants\%20for\%20the\%20Layman.pdf.

[10] TTRL Report of Room Temperature Crush Resistance Tight ness Tests on Selco 4" Class 150 lb SS316 Gasket [OL]. http://www.selcoseal.com/CrushResistance.pdf.

[11] Fig.3-8 O-Ring Leak Rate, Parker O-Ring Handbook ORD 5700 http://www.parker.com/literature/ORD\%205700\%20Parker_O -Ring_Handbook.pdf 


\section{Annex A Further Dissections of the Prior Art}

\section{A.1 Garlock Helicoflex Idea for over 60 years (see references [8]) that is closest to Xu's sealing difficulty factor $\boldsymbol{m}_{2}$}

As shown in table A.1 and Fig.A.1, Garlock Helicoflex has realized that a sealing element:

- should be designed to have a soft outer layer and a strong substrate so that the substrate can provide enough specific load

to plastically deform the soft outer layer into the flange imperfections, and

- should be assembled (compressed) to the point $\left(\mathrm{e}_{2}, \mathrm{Y}_{2}\right)$ with full plastic and elastic deformations so as to create and maintain an optimum sealing contact (see the load-deflection curves in table A.1);

but has not yet realized that the elastic deformation from the ring substrate is useful and the elastic deformation from the ring outer layer is not useful, or has not yet realized that the soft and elastic nature of rubber causes its sealing element to be the most difficult to create a leak-free connection, and so has rested at the level to simply copy the softness and elasticity of rubber O-rings.

Therefore, the difference between Garlock Helicoflex idea and the sealing difficulty factor is that between the perceptual knowledge and the science. However, there is no initial perceptual knowledge as a basis, and no final science as a result.

Table A.1 Spring Energized Metal O-Rings
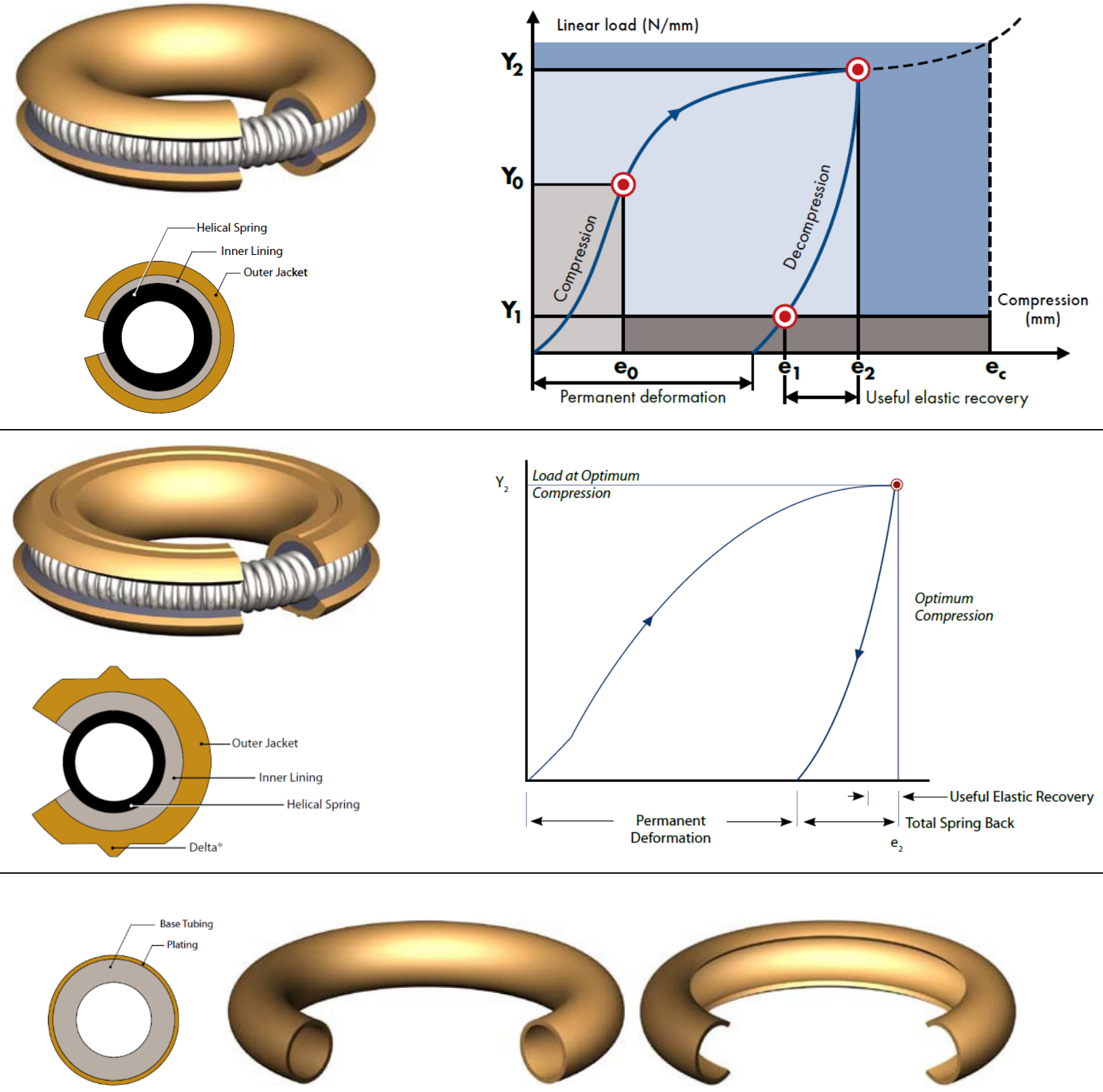

Fig.A.1 Metal O-Rings and C-Rings with a Soft Plating layer 


\section{A.2 Prior art gaskets that are inadvertently closest to the designing concept of a both soft and strong seal}

As shown in Fig.A.2, spiral wound gaskets are made by spirally winding a formed narrower metal strip and a soft narrow filler layer; Kammprofile gaskets are made by adding a soft material layer on each face of a serrated metal core ring; and solid metal core gaskets are made by adding a soft material layer on each face of a solid metal core ring (see Flexitallic Gasket Design Criteria). Though the sealing difficulty factors $m_{l}, m_{l}=$ the contact layer's modulus $\left(E_{c}\right) /$ the substrate's modulus $\left(E_{s}\right)$, for the three gaskets are outwardly less than one, their tightening design and method

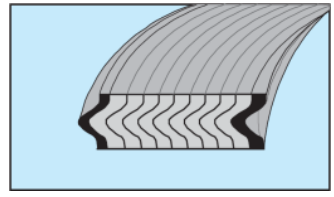

Fig.A.2a Spiral Wound Gasket

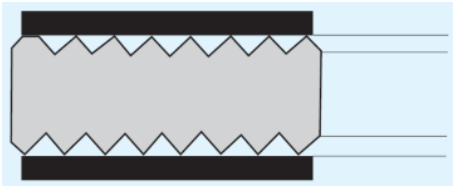

Fig.A.2b Kammprofile Gasket cannot cause such a tightened deformation with its circumferential uniformity within $\mu \mathrm{ms}$ that their soft outer layers in a certain orientation are so thicker as for their local sealing difficulty factors $m_{1}$ to be still one; and hence they have a sealing-stopping stress $\left(G_{s}\right)$ whose average is far less than $0.1 \mathrm{MP}_{\mathrm{a}}$ and have an assembled stress $\left(S_{a}\right)$ whose average is very great, but cannot be assembled for all the time up to a fully tight contact in each orientation or they have no vertical leak-free segment in their working curves.
As shown in Fig.A.3 (see References [10]), the sealing contact rigidity of a Selco gasket is determined by the Young modulus $E$ of its soft sealing material in grooves and can be always very low. However, in its Crush Resistance Tightness Test, the incompressibility of its soft sealing material encapsulated in grooves can cause its supporting rigidity to be high up to the material rigidity of its grooved metal carrier after it is compressed to reach its fully plastic and elastic deformations, and its metallic face can improve but not fully ensure its circumferentially uniform contact, so that its stress calculated for a required leakage rate to approach but not to equal its actual stress. Accordingly, its working line approaches but is not yet a vertical line.

Therefore, it can be said that the prior art has fully proved Xu's designs and parameters for sealing elements correct.
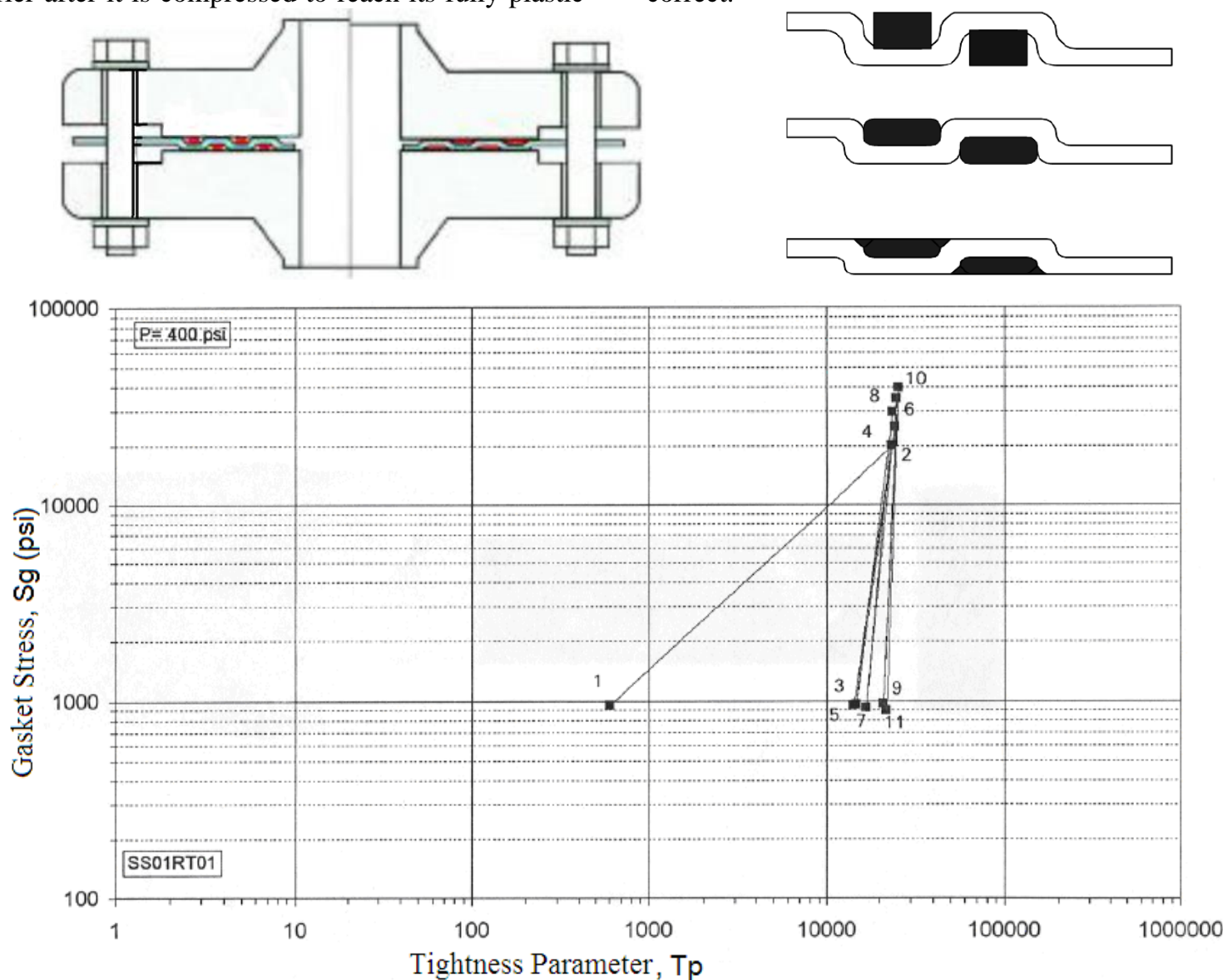

Fig.A.3 Crush Resistance Tightness Tests on Selco 4" Class 150 lb SS316 Gasket 